\title{
Data Storage Integrity Checking with Efficient Privacy Preserving Model for Cloud
}

\author{
K. T. Subhadra ${ }^{1}$, S. S. Jaya ${ }^{2}$ \\ ${ }^{1}$ Associate Professor, Sri Sai Ram Engineering College \\ ${ }^{1}$ Research Scholar, Anna University, Chennai \\ ${ }^{2}$ Director-Research, Adhiparasakthi Engineering College, Melmaruvathur
}

\begin{abstract}
Cloud computing is a growing technology that offers compute, storage and network resources as a service over the internet. It enables the individuals, clients or the enterprises to outsource their data and application software to the cloud server. The services are offered by a cloud service provider (CSP) and the users need to pay for what they use. There are many security concerns needs to be addressed when the data is maintained by third party service provider in cloud. The auditor is introduced to audit the integrity of the data on behalf of the client in order to ensure integrity of data. This can be called as public auditability of data. Recently, two privacy preserving auditing mechanisms named Oruta and Knox are introduced to check the correctness of stored data. In this paper, we try to propose the security flaw of their scheme when active adversaries are involved in cloud storage. An active adversary is capable of modifying the data stored in cloud arbitrarily. This data modification is not being identified by the user and the auditor in the verification process. We try to suggest a solution to resolve this flaw by signing the proof response generated on the cloud server side. Then the signed proof is sent to the trusted third party auditor (TTPA) for verification. The auditor first verifies the signature and for the validation of the proof. The proposed scheme is proved to be secure against active adversary.
\end{abstract}

Keywords - Auditing, cloud storage, data, integrity, privacy preserving, TTPA, Security

\section{INTRODUCTION}

Cloud computing as the definition given by National Institute of Standards and Technology (NIST) [1], is "an enabling ubiquitous model, provides us network access based on demand and offers a convenient aid of collection of configurable resources like servers, networks and storage applications, various services, which has the ability to be quickly provisioned and released with minimum effort from service provider interface or minimal management effort [1]." Cloud computing is based on accessing resources via the Internet.

The three common cloud service models offered by cloud is software as a service (SaaS), Infrastructure as a service (IaaS) and Platform as a service (PaaS). Out of this, Cloud storage is the main important service offered by cloud computing, it helps users to move their data in to the cloud from local storage systems. It is an easy and cost effective way to store and manage the data. Drop box and GoogleDocs is an example of cloud storage system [3] and it now becomes the essential feature in storage offerings.

Providing security for data is a major concern in cloud storage systems even though it comes with attractive benefits $[1,2]$. The internal and external threats cause the data in cloud to be deleted or corrupted or tampered $[4,5]$. In specific any external adversary tries to alter the content of the stored data and convinces the owner of the data that their data stored in cloud is correct and intact [6]. This is being done for high profit [7]. Hence it becomes essential to verify the correctness and integrity of the outsourced data moved to cloud.

\section{RELATED WORK}

Several schemes and auditing protocols have been introduced for protecting the integrity of outsourced data [817]. Two schemes PDP [8] and POR [9] were introduced initially to cross check the integrity of outsourced data. They followed public auditing method on RSA based homomorphic linear authenticators. Ateniese et.al [10] provided scalable PDP to work with dynamic datas such as insertion, updation and deletion based on Hash functions and encryption technologies. It does not allow block insertion and has a fixed bound on no of challenges generated.

Erway [11] and Jules [9] provided dynamic auditing protocols to guarantee possession of data and data is retrievable. They do not consider the case of updation operation. Shacham and waters [12] proposed a solution for the above based on pseudorandom functions, BLS signature and their protocol supports public auditing. T.subha and S.Jayashri [13] described a method to perform dynamic data operations and their scheme supports public auditing by introducing TTPA (trusted third party auditor) in hybrid cloud. Wang et al described methods to achieve storage correctness, public auditability, privacy-preserving, batch auditing, error localization, error recovery and dynamic data operations. 
In all the above schemes the cloud user delegates the integrity checking process to a third party auditor (TPA). An auditor is capable and trusted entity is allowed to audit the integrity of the outsourced data in cloud server. Existing schemes mostly focused on verifying the integrity of the data stored in remote servers.

Wang et.al [14-16] focuses on privacy preserving, which is an important property during the verification process. An adversary alters the cloud data arbitrarily and is able to provide a strong and valid proof in order to pass the verification process.

Adversary fools the auditor by generating valid proof and ensures the data owner to believe the data is well maintained and stored by the cloud server and integrity is preserved. Actually the data has been corrupted by the adversary [17].

Adversary needs to remember only how the data is altered, do not need to register the tags or content of the data. In this paper, we try to suggest a solution to resolve this problem by maintaining the features of original scheme proposed in Oruta [15].

\section{Preliminaries}

We revise some basic terminologies used in the auditing schemes and the system model used in the schemes.

\subsection{Bilinear maps:-}

Let $G_{1}, G_{2}$ and $G_{T}$ are denoted as multiplicative cyclic groups. Let $\mathrm{p}$ is a prime number and generators of G1 and G2 are g1 and g2. The map is e: G1xG2 said to be a bilinear mapping if it holds the following conditions [2-7].

a) e is bilinear for all $a, b \in Z p$

b) $\mathrm{e}$ is non-degenerate, i.e. $\mathrm{e}(\mathrm{g} 1, \mathrm{~g} 2) \neq 1 \mathrm{GT}$

c) is efficiently computable.

\subsection{System Model:-}

Three entities are basically involved in the scheme discussed below. The three entities are

- Users (or) owner of data

- $\quad$ Trusted third party auditor (TTPA), which is newly introduced in our system and

- $\quad$ Cloud service provider(CSP)

are present in the auditing system.

Users has large amount of data to be outsourced into cloud. We assume the trusted third party auditor (TTPA) is a trusted one and has the capabilities to perform auditing process based on user's request. Cloud service provider offers storage space to the users. The system model is represented in Fig.1.

The auditing request is generated by the auditor based on the request from user. The auditor sends the challenged blocks to the service provider. CSP in turn generates the proof for the challenged blocks and responds to the request.

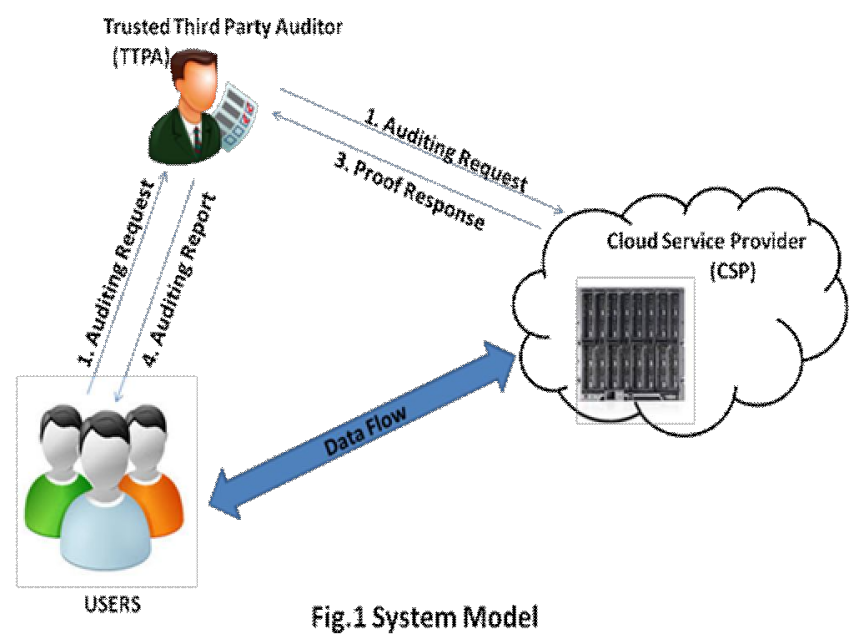

Then the auditor (TTPA) runs the algorithm to verify whether the proof passes the integrity verification or not. It outputs TRUE, if it passes the verification process otherwise outputs FALSE. Finally the detailed auditing report is sent back to the user. We have explained our system named data integrity verification in hybrid cloud using TTPA in our previous paper [13]. Our previous version of the paper described the auditing process carried out using TTPA. The key points are discussed below.

\subsection{Public auditability of data:-}

Our scheme consists of three important phases on the client side. They are, Initialization phase, Key Generation phase and Tag Generation phase:

\section{a) Initialization phase:}

Initially preprocessing of file is being done. The signatures are added to the corresponding file blocks by running the algorithm. The original file, file tag and the set of signatures are moved to the cloud. It is deleted from local storage. The Meta data used for verification is shared with the auditor (TTPA) to execute the integrity check. 
Step1: The original file to be outsourced is divided into small blocks

$\mathrm{F}=\{\mathrm{b} 1, \mathrm{~b} 2$, bn $\}$ for $b_{i}(i=1 \ldots . n)$

\section{b) Key Generation phase:}

Step 2: The pair of keys (Private key (or) Secret key - SEK and Public key - PUK) are generated. If a client wants they can encrypt the data using a secret key (SEK) before outsourcing. This enables us to provide confidentiality for the data to be outsourced. Random element $r$ is chosen and the public key is calculated by $\mathrm{v} \leftarrow \mathrm{g}^{\mathrm{r}}$. The public key is given as $(\mathrm{v}, \mathrm{PUK})$ and the private key is (r, SEK).

\section{c) Signature Generation phase}

Step 3: File tag is generated to identify a file. File tag contains the name of the file and random value.

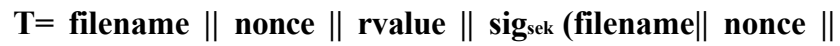
rvalue)

Step 4: Finally the signature is generated for the file blocks based on the secret key SEK. As given by Wang et. al [14].

We adopt a Merkle Hash Tree(MHT) structure to store the tags. MHT is a authentication structure used to authenticate data blocks.

Fig.2. Merkle Hash Tree

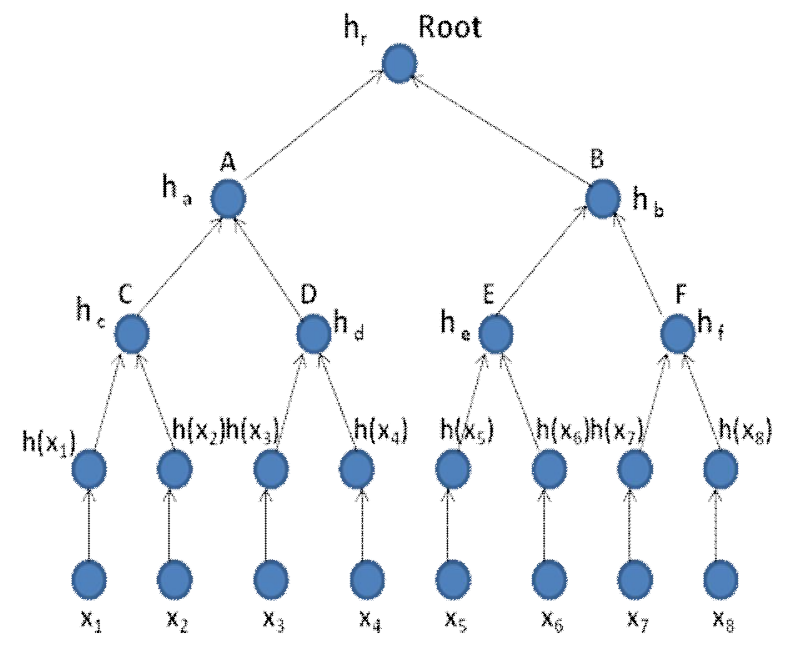

A signature $\Omega_{\mathbf{i}}$ ( generating hash value by applying hash algorithm) computed for all the data blocks from $\{\mathrm{b} 1, \mathrm{~b} 2$, bn $\}$.

$$
\mathbf{\Omega}_{\mathbf{i}}=\left(\mathbf{H}\left(\mathbf{b}_{\mathbf{i}}\right) . \mathbf{u}^{\mathbf{b i}}\right)^{\mathbf{r}}
$$

Signature set for all the blocks is given by,

$$
\Pi=\{\Omega \mathbf{i}\}, \mathbf{1} \leq \mathbf{i} \leq \mathbf{n} \text {----------- (4) }
$$

Using secret key the root $\left(\operatorname{sig}_{\text {sek }}(\mathrm{H}(\mathrm{R}))^{\mathbf{r}}\right.$ of the MHT is signed. The above steps are performed on the client's side.

Then the following data $\left(F, \boldsymbol{\Pi},\left(\operatorname{sig}_{\text {sek }}(H(R))^{\mathbf{r}}\right)\right.$ is outsourced onto the cloud provider. It is deleted from the local storage.

\subsection{Data auditing Mechanism:-}

The auditing of data is outsourced to a trusted third party auditor (TTPA). TTPA is the one, who is expertise and have the capabilities to perform auditing based on the request from client. Auditor can anytime challenge the server for the integrity of the data. Then it checks and prove the response from the server is a valid one.

Our scheme consists of two important phases in the second stage.

\section{a) Challenge responsephase}

Step 1: Auditor sends a challenge request for the randomly selected blocks. Auditor also specifies the block positions to be checked by server. Challenge message contains the number of queried blocks. It randomly picks the element from the subset $\mathrm{S}=\left\{\mathrm{S}_{1} \leq \ldots \leq \mathrm{Sc}\right\}$ of set belongs to $[1, \mathrm{n}]$. Auditor sends the challenge message to the cloud server.

$$
\text { Chal msg }=\left\{\left(\mathbf{i}, \mathrm{m}_{\mathrm{i}}\right)\right\} \mathrm{s} 1 \leq \mathrm{i} \leq \mathrm{sc}
$$

Step 2: Server takes an input file $F$, challenge request query and the Signature set $\mathrm{S}$ after receiving the challenge request generated by TTPA. He sends the proof as the response to the auditor.

Server sends data proof and the signature proof as follows.

$$
\mathbf{P}=\sum_{i=s 1}^{s c} v_{i} b_{i} \text { and } \boldsymbol{\Omega}_{\mathbf{i}}=\prod_{i=s 1}^{s c} \Omega \mathrm{i} \mathrm{v}^{\mathrm{i}}
$$

\section{b) Proof Verification phase}

Step 3: Auditor (TTPA) checks for integrity after receiving the response by running the algorithm. As a next step, newly computed signature is compared with the previously stored signature.

$$
\mathbf{e}(\Omega, \mathbf{g}) \stackrel{?}{\Leftrightarrow} \mathbf{e}\left(\prod_{i=s 1}^{s c} \mathrm{H}(\mathrm{bi})^{\mathrm{vi}}, \mathrm{u}, \mathrm{r}\right)
$$


Step 4: It outputs TRUE if both are same otherwise FAIL.

Step 5: The audit result is sent to the data owner.

\section{Privacy Preserving method}

As we have already pointed out that the data returned from server is susceptible to attacks from active adversaries $[3,15,16,17]$. The adversaries are able to modify or alter the content of the data without the knowledge of the auditor. The auditor believes that the response given by server is a valid and authenticated one.

The cryptanalysis of these protocols were studied and pointed out by Wang et.al $[15,16]$ and Yong Yu et al [3]. The proposed auditing mechanism is exposed to security attacks when active adversaries involved. The reason is there is no method to prove the authenticity of the response sent by the server. In this paper, we recommend an improved suggestion to preserve (or maintain) the privacy of data in cloud server based on the security flaw discussed by Wang and Yongyu.

Our scheme is able to detect the corrupted data by an active adversary. Our auditing protocol uses digital signature algorithm in association with certificates. This certificate is used to check the authenticity of server. Certificates bind identity information like user name, ipaddress and social security number (SSN) to a public key with the help of digital signatures. Hence, no one is able to access the data.

We try to achieve a solution without compromising on original scheme features. Our system incurs low computation cost since it preserves the properties of original scheme.

\subsection{Our Improved Suggestion:-}

Our scheme is discussed in detail below.

\section{Step1:}

Initially in KeyGen phase, the user (or) data owner applies for a certificate from CA (Certificate Authority) by giving his identity, public key.

CA issues the certificate in the below format. It contains,

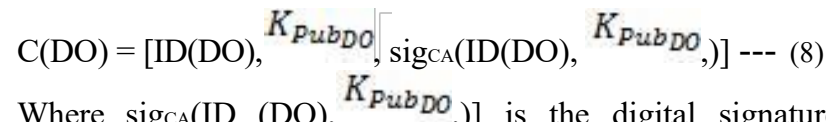
generated by Certificate Authority using its secret algorithm.

\section{Step 2:}

Data owner verifies the authority using verification algorithm verCA[C (DO)].

\section{Step 3:}

Then this certificate must be communicated securely to TTPA and Cloud server. There may be a possibility that adversary can act as a legitimate user, and then he may get a valid certificate that contains public key and identity information. In order to avoid this, the user distributes certifying authorities' verification algorithm verCA $[\mathrm{C}(\mathrm{DO})]$ to the auditor and cloud server. Cloud server has also received his certificates.

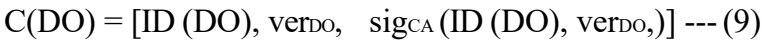

$$
\begin{aligned}
& \mathrm{C}(\mathrm{CS})=\left[\mathrm{ID}(\mathrm{CS}), \text { vercs } \operatorname{sig}_{\mathrm{CA}}\left(\mathrm{ID}(\mathrm{DO}), \operatorname{ver}_{\mathrm{Cs}},\right)\right]-----(10)
\end{aligned}
$$

\section{Step 4:}

A secure authentication channel is established using a session key while communicating between TTPA and cloud server. This session key is shared between these two parties. Cloud server signs the generated proof $(\mathrm{P})$ using the secret key $(\mathrm{SK})$ and creates signature $(\sigma)$ on it. Then it sends $(\mathrm{P}, \sigma, \mathrm{C}$ (DO)) to the TTPA.

\section{Step 5:}

As a first step, upon receiving the signature TTPA first checks the Certificate using its verification algorithm. If it is proved, then the response is verified using its public key (PK). Otherwise the integrity verification is not continued.

\section{Step 6:}

As a second step, the proof response is further decrypted using public key (PK). This is done after the certificate is verified for checking authenticity. Then the signature is verified to prove the authentication of server.

\section{Step 7:}

Finally TTPA validates the proof by running the verification algorithm. That's how the data is protected from any kind of modification from any active adversaries.

\section{RESUlTS AND DiscuSSIONS}

Eucalyptus software has been used to conduct our experiments. This tool helps us to explore the private cloud setup and various functionalities. This eucalyptus platform can be used to experiment IaaS related services. We have installed eucalyptus fast start version 3.4.1 on Centos6 in an Intel core i5-3520 CPU at $2.2 \mathrm{GHz}, 500 \mathrm{~GB}$ SATA drive and $8 \mathrm{~GB}$ RAM. Our algorithms have been implemented in this setup 
and the results are discussed here. The results are shown inthe Fig. 3, Fig. 4 and Fig. 5.

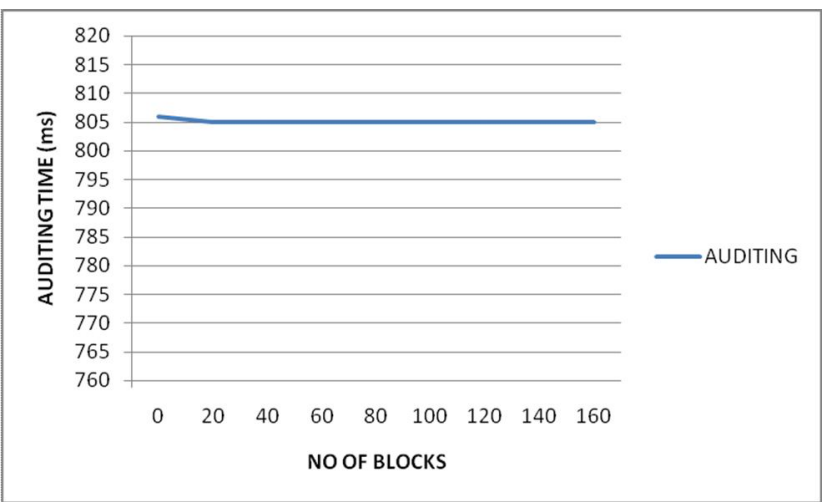

Fig.3 Performance of Auditing Time Vs No of Blocks

The above graph shows the time taken to audit the no of blocks. Block size ranges from small to large and is given in KB. The auditing time maintains a probability of above $95 \%$ irrespective of block size.

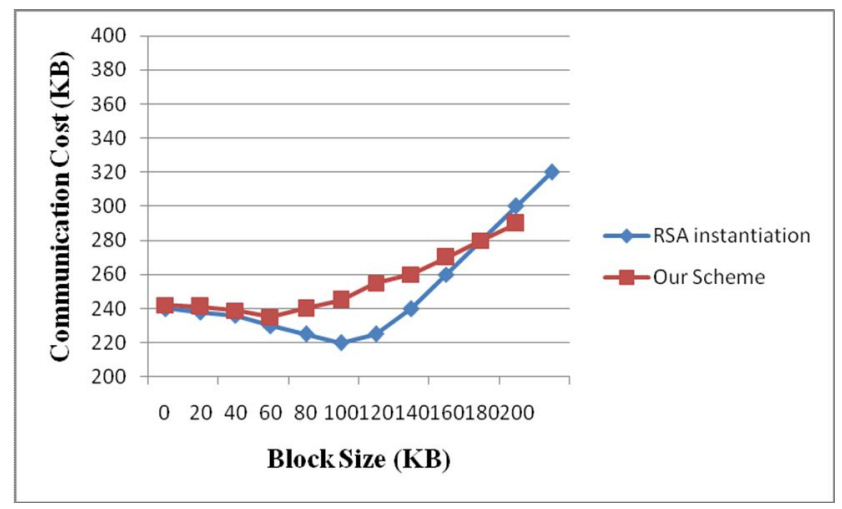

Fig.4 Communication Cost Vs Block Size

The above graph is plotted between the size of the blocks and the communication cost incurred during auditing by sending and responding with proofs. As compared with the existing scheme [14], our scheme tries to achieve the minimum probability. The communication cost increases linearly as the no of blocks grows in size.

The privacy performance of our proposed system is shown in Fig.5. Our method maintains the privacy of users of above 95\% when active adversaries are involved in the system. Our system is able to detect the changes in the stored data.

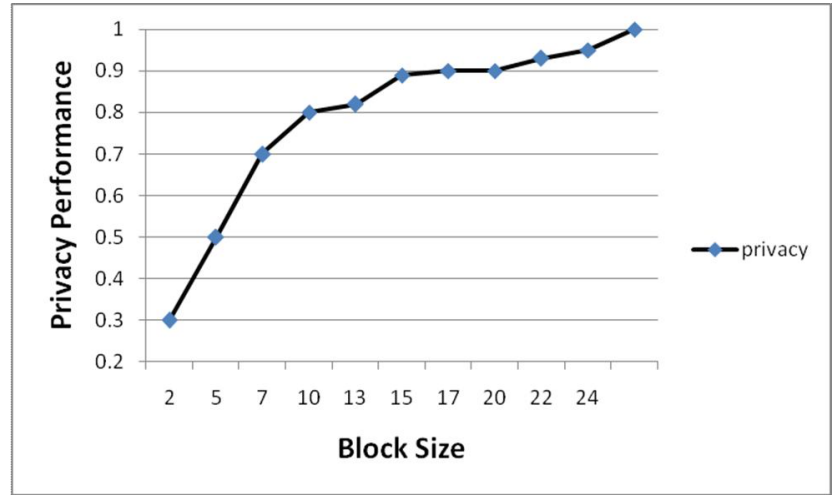

Fig.5. Privacy Performance

\section{CONCLUSION}

In this paper, we proposed a solution to protect the privacy of a user data from active adversaries. We provided a technique to sign the data using digital signature algorithm in association with certificates. We established a secure authentication channel between TTPA and cloud server while sending and receiving challenges and responses. It enables us to protect the data from external adversary. Our scheme preserves the properties of original scheme.

\section{REFERENCES}

[1]. P.Mell and T.Grance, "The NIST Definition of Cloud Computing", NIST Special Publication - 800145, 2011; http://csrc.nist.gov/publication/nistpubs/800-145.

[2]. M.Lillibridge, S.Elnikety, A.Birrell, M.Burrows and M.Isard, "A Cooperative Internet Backup Scheme," proc. USENIX Ann.Technical Conf., pp.29-41, 2003.

[3]. Yong.Yu, Lei Niu, Guomin Yang, Yi Mu, Willy Susilo, "On the security of auditing mechanisms for secure cloud storage", Future Generation Computer Systems 30(2014), 127-132.

[4]. Lee Cheng-Chi, Lai Yan-Ming, Hsiao Chin-Sung, Cryptanalysis of a simple key assignment for access control based on polynomial. J Inf Secur Appl 2013; 18(4):215-8.

[5]. Li H, Dai Y, Tian L, Yang H, “ Identity-based authentication for cloud computing, Lecture Notes of Computer Science (LNCS), vol. 5931; 2009, p.157-166.

[6]. Tadapaneni, N. R. (2016). Overview and Opportunities of Edge Computing. Social Science Research Network.

[7]. Yuan Zhang, Chunxiang $\mathrm{Xu}$, Jining Zhao, Xiaojun Zhang, Junwei Wen, "Cryptanalysis of an Integrity checking scheme for cloud data sharing", Journal of Information Security and applications (2015), I-6.

[8]. Dolev Danny, Yao Andrew C, "On the security of public key protocols. Inf Theory, IEEE Trans Inf Theory 
March, 1983; 29(2):198-208.

[9]. G.Ateniese, R.Burns, R.Curtmola, J.Herring, L.Kissner, Z.Peterson, and D.Song, "Provable data possession at untrusted stores,"in Proc.of CCS'07. Newyork, NY, USA: ACM, 2007, pp.598-609.

[10]. Tadapaneni, N. R. (2017). Different Types of Cloud Service Models. Available at SSRN 3614630.

[11]. G.Ateniese, R.D.Pietro, L.V.Mancini, G.Tsudik, "Scalable and efficient provable Data possession," in: Proc. of SecureComm 2008, pp. 1-10.

[12]. C.C.Erway, A.Kupcu, C.Papamanthou, R.Tamassia, "Dynamic provable data possession," Proc. of CCS

2009, pp.213-222.

[13]. H.Shacham and B.Waters, "Compact proofs of retrievability," in Proc. of ASIACRYPT'08. Melbourne, Australia: Springer-Verlag, 2008, pp.90-107.

[14]. T.Subha and Dr.S.Jayashri, "Data Integrity Verification in hybrid cloud using TTPA," Lecture Notes in Electrical Engineering 284,Springer, pp 149- 159.

[15]. Q.Wang, C.Wang, J.Li, K.Ren, and W.Lou, "Enabling public verifiability and data dynamics for storage security in cloud computing," in Proc. of ESORICS'09. Saint Malo, France: SpringerVerlag, 2009, pp.355-370.

[16]. B.Wang, B.Li, H.Li, "Oruta: privacy preserving public auditing for shared data in the cloud," in: IEEE International Conference on Cloud Computing, 2012, pp.293-302.

[17]. B.Wang, B.Li, H.Li, "Knox: privacy preserving auditing for shared data with large groups in the cloud," in: Proc. of ACNS 2012, pp.507-525.

[18]. Jianbing Ni, Yong Yu, Yi Mu, Qi Xia, "on the security of an efficient dynamic auditing protocol in cloud storage," IEEE Trans on Parallel and Distributed Systems, Vol.25, No.10, October 2014.

[19]. A.Juels and B.S.Kaliski, Jr.,'Pors: proofs of retrievability for large files," in Proc.of CCS'07. Newyork, NY, USA: ACM, 2007, pp.584-597.

[20]. Abraham, W. K., Saravanan, S., \& Smith, K. (2019, June). Security and Privacy Challenges in Fog Computing. In International Conference on Fog Computing. 